\begin{tabular}{|c|c|}
\hline \multirow{3}{*}{ 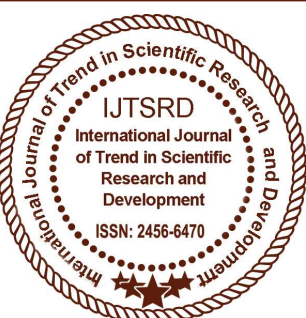 } & $\begin{array}{l}\text { International Journal of Trend in Scientific } \\
\text { Research and Development (IJTSRD) }\end{array}$ \\
\hline & International 0 \\
\hline & ISSN No: 2456 - 6470 | www.ijtsrd.com | Volume - 2 | Issue - 5 \\
\hline
\end{tabular}

\title{
NSGA-II Implementation for Resource Allocation in MIMO-OFDMA
}

\author{
P. Kanthimathi \\ Lecturer Senior Grade, Nachimuthu Polytechnic College, \\ Pollachi, Tamil Nadu, India
}

\begin{abstract}
One of the most challenging issues for future wireless communication systems is the provision of quality-ofservice (QoS) guarantees to users over the harsh wireless channels given the limited resource availability. The aim of this paper is to probe the problem of dynamic subcarrier and bit allocation in downlink of MIMO-OFDMA systems. Using Singular Value Decomposition, MIMO fading channel of each subcarrier is transformed into an equivalent bank of parallel SISO sub-channels. NSGA-II is handled which is a multi-objective Genetic Algorithm, for joint allocation of bits and subcarriers, in the downlink of MIMO-OFDMA system. NSGA-II is intended for optimization problems involving multiple conflicting objectives. Here the two conflicting objectives are Rate Maximization and Transmit Power Minimization.
\end{abstract}

Keywords: MIMO-OFDM, Non-dominated Sorting genetic algorithm, Multi-objective optimization, Elitism, Singular value decomposition

\section{INTRODUCTION}

Wireless communications has emerged as one of the largest sectors of the telecommunications, industry, evolving from a niche business in the last decade to one of the most promising areas for growth in the recent era.

The increasing demand for wireless multimedia services are in need of reliable and high-rate data communications over a wireless channel. High channel capacities or transmission rate for the same bandwidth with no additional power requirements by employing multiple antennas (MIMO) at the transmitter and receiver is contributed by Spatial multiplexing.(OFDM) efficiently uses the spectrum by spacing the channels closer, and it also has the ability to reduce ISI. The blend of these two technologies has been researched for the most hopeful applicant technique for the next generation wireless systems. Due to the frequency-selective fading, a major portion of the subcarriers assigned to a specific user would undergo severe fading and power will not be efficient to carry any data. As the number of user increases the Multiuser system creates channel. Adaptively assigning resources to each user provide a better performance compared to fixed schemes and it is based on their channel condition which can, abuse multiuser diversity [2].

The MIMO/OFDM system enjoys more degrees of freedom for efficient resource allocation and it is due to the spatial parallelism and frequency selectivity of the channel.

\section{MIMO-OFDMA}

Multi-user MIMO-OFDMA is regarded as an important technology for increasing the flexibility and efficiency of wireless communication systems [2].In the MIMO-OFDMA downlink system model from the base station (BS), all channel state information of each transmit and receive antennas is sent to the subcarrier and power allocation block through the feedback channels from all mobile users. The resource allocation scheme is forwarded to the MIMO-OFDM transmitter. The transmitter then loads each user's data onto its allocated subcarriers.

The resource allocation scheme is updated as soon as the channel information is composed and also the subcarrier and bit allocation information is guided to each user for the purpose of detection, through a separate channel [6]. 
MIMO exploits the space dimension to progress wireless systems capacity, range and consistency. It offers significant escalations in data throughput and link range without supplementary bandwidth or increased transmit power. MIMO achieves this goal by spreading the same total transmit power over the antennas to achieve an array gain that progresses the spectral efficiency (more bits per second per hertz of bandwidth).

\section{SYSTEM MODEL}

The system under deliberation is downlink of a MIMO-OFDMA system, with $\mathrm{K}$ users and $\mathrm{N}$ subcarriers. At the transceiver the perfect channel state information is assumed. Then each subcarrier composed of a narrowband channel with $\mathrm{M}$ antennas at both the receiver as well as the transmitter, which can be modeled by $\mathrm{M} \times \mathrm{M}$ channel matrix and it is of $\mathrm{H}=[\mathrm{hi}, \mathrm{j}]$, where hi,j is the gain of the channel at the receive antenna $i$ from transmit antenna $j$. The SVD of $\mathrm{H}$ is then written as:

$$
\mathrm{H}=\mathrm{U} \wedge^{1 / 2} \mathrm{~V}^{\dagger}
$$

Where $\mathrm{U}$ and $\mathrm{V}$ are the unitary matrices, $\mathrm{V}^{\dagger}$ denotes the transpose conjugate of $V$, and $\Lambda$ is a diagonal matrix. The elements of $\Lambda=\operatorname{diag}\left(\left[\lambda_{1} \ldots . \lambda_{M}\right]\right)$ are real and they are arranged as $\lambda_{1} \geq \ldots \geq \lambda_{M} \geq 0$.The SVD analysis is shadowed by the breakdown of channel matrix $\mathrm{H}$ into a number of self-governing orthogonal excitation modes, and it is known as eigen-modes of the channel.

The transmit preceding and receiver shaping convert the MIMO channel into $R_{H}\left(R_{H}\right.$ is the rank of the matrix H) parallel SISO channels with input $\tilde{x}$ and output $\tilde{y}$

Since from the SVD, it has,

$$
\tilde{y}_{\mathrm{m}}=\sqrt{\lambda_{\mathrm{m}}} \tilde{\mathrm{x}}_{\mathrm{m}}+\tilde{\mathrm{n}}_{\mathrm{m}}, \text { form }=1, \ldots \mathrm{M}
$$

Here $\tilde{n}=U^{\dagger} n$, and $n$ denotes to Additive White Gaussian Noise(AWGN).

In the frequency selective fading different subcarrier constitute of different MIMO channels. The MIMO channel matrix $H_{k, n}$ for the $k_{t h}$ user and the $n_{t h}$ subcarrier is disintegrated into $\mathrm{M}$ parallel SISO channels, where channel gains are given by

$$
\sqrt{\lambda_{\mathrm{k}, \mathrm{n}, \mathrm{m}}}\left(\lambda_{\mathrm{k}, \mathrm{n}, 1} \geq \ldots \geq \lambda_{\mathrm{k}, \mathrm{n}, \mathrm{M}} \geq 0\right) \text {. }
$$

\section{OPTIMIZATION PROBLEM}

The transmit power for the $\mathrm{k}_{\mathrm{th}}$ user and the $\mathrm{m}_{\mathrm{th}}$ Eigenmode of the $n_{\text {th }}$ subcarrier must be equal to:

$$
\mathrm{P}_{\mathrm{k}, \mathrm{n}, \mathrm{m}}=\mathrm{f}_{\mathrm{k}}\left(\mathrm{c}_{\mathrm{k}, \mathrm{n}, \mathrm{m}}\right) / \lambda_{\mathrm{k}, \mathrm{n}, \mathrm{m}}
$$

Where $c_{k, n, m}$ is the bits allocated for unit channel gain. The overall transmit power is given by

$$
\mathrm{P}_{\mathrm{T}}=\sum_{\mathrm{k}=1}^{\mathrm{k}} \sum_{\mathrm{n}=1}^{\mathrm{N}} \rho_{\mathrm{k}, \mathrm{n}} \sum_{\mathrm{m}=1}^{\mathrm{M}} \frac{\mathrm{f}_{\mathrm{k}}\left(\mathrm{c}_{\mathrm{k}, \mathrm{n}, \mathrm{m}}\right)}{\lambda_{\mathrm{k}, \mathrm{n}, \mathrm{m}}}
$$

Where the subcarrier allocation indicator $\rho_{k, n}$ is defined as

$\rho_{\mathrm{k}, \mathrm{n}}$

$= \begin{cases}1, & \text { if the } n \text {th subcarrier is allocated to kth user } \\ 0, & \text { otherwise }\end{cases}$

Problem is framed to maximize the minimum data rate among entire users subject to the constraint that the total power cannot exceed a given value. The power objective is modified little and assumes that the total available transmission power is limited to a certain range with a typical value $\mathrm{P}_{\mathrm{T}}$.Second objective to bring the total power as close as possible. The multi-objective optimization problem is:

$$
\begin{aligned}
& \underset{c_{k, n, m}, \rho_{k, n}}{\operatorname{man}} \min _{k} R_{k}=\max _{c_{k, n, m, \rho_{k, n}}} \min _{k} \sum_{n=1}^{N} \sum_{m=1}^{M} c_{k, n, m} \rho_{k, n} \\
& 6-\operatorname{cin}_{c_{k, n, m}, \rho_{k, n}}\left|P_{T}-\sum_{k=1}^{N} \sum_{n=1}^{N} \rho_{k, n} \sum_{m=1}^{M} \frac{f_{k}\left(c_{k, n, m}\right)}{\lambda_{k, n, m}}\right|
\end{aligned}
$$

Subject to constraints:

$$
\begin{aligned}
& C_{1}: \sum_{K=1}^{K} \mathrm{R}_{\mathrm{K}} \leq \mathrm{NMD}_{\max } \\
& C_{2}: \sum_{\mathrm{K}=1}^{\mathrm{K}} \rho_{\mathrm{k}, \mathrm{n}}=1 \text {, and } \rho_{\mathrm{k}, \mathrm{n}}=\{0,1\}
\end{aligned}
$$

$\mathrm{R}_{\mathrm{K}}$ denotes the transmit bits per OFDM symbol for the $\mathrm{k}_{\text {th }}$ user, $\mathrm{D}$ is a set of available bits in the adaptive modulation, $\mathrm{D}_{\max }$ denotes the maximum value in the set $\mathrm{D}$. Constraint $\mathrm{C}_{2}$ takes care of the fact that one user is assigned with one subcarrier only.

\section{NSGA-II}

Non-dominated Sorting Genetic Algorithm is to use to progress the adaptive fit of a population of candidate solutions to a Pareto front which is inhibited by a set of objective functions [9]. The algorithm uses an 
evolutionary process with for evolutionary operators that include selection, genetic crossover, and genetic mutation. The population is sorted into a hierarchy of sub-populations based on the ordering of Pareto dominance. Similarity between members of each subgroup is evaluated on the Pareto front, and the resulting groups and similarity measures are used to endorse a various front of non-dominated solutions. NSGA-II algorithm includes the following steps to be employed:

\subsection{Population initialization}

The number of individuals in the population $P$ and the number of generations $\mathrm{G}$ are initialized and can be varied for different runs of the algorithm. Each individual is created by generating 0 's and 1's randomly.

\subsection{Evaluate the objective functions}

For each individual the fitness values of the objective function are calculated.

\subsection{Non-dominated sorting}

Based on non-domination the population is sorted into fronts and each individual is assigned a rank. After the completion of non-dominated sorting the crowding distance is assigned.The non-dominated sorting algorithm is done by following steps:

Step 1: From the main population $\mathrm{P}$ each individual $\mathrm{p}$ is made to do the following:

- Initialise $\mathrm{Sp}=\phi$

\{set of individual being dominated by $\mathrm{p}$ \}

- Initialise $\mathrm{Np}=0\{$ no of individual that dominates p\}.

- Consider individual $\mathrm{p}$ in population $\mathrm{P}$

$>$ If $\mathrm{p}$ dominates $\mathrm{q}$ then $\mathrm{S}_{\mathrm{p}}$ is

$\mathrm{S}_{\mathrm{p}}=\{\mathrm{q} \mathrm{U} \mathrm{Sp}\}$

$>$ Else $\mathrm{Np}=\mathrm{Np}+1$

Step 2: If $\mathrm{Np}=0$ (no individual dominates $\mathrm{p}$ and it belongs to first front).Set $p_{\text {rank }}=1$.First front update is made with addition of $\mathrm{p}$ to the $\operatorname{set}\left(\mathrm{F}_{1}=\{\mathrm{p} \mathrm{U} F i\}\right)$.

Step 3: Intialize front counter $\mathrm{i}=1$

(Carry out for the entire individual in main population

P)

- For each individual q in $\mathrm{Sp}$

$>\mathrm{Nq}=\mathrm{Nq}-1(\mathrm{Nq}$ is domination count)

$>$ If $\mathrm{Nq}=0$ then

$>\mathrm{q}$ rank $=\mathrm{i}+1$

- update $\mathrm{Q}=\{\mathrm{Q} \mathrm{U}$ q $\}$
Step 4: If Fi not equal to 0 then

$\mathrm{Q}=0$ \{set for storing individual in $\mathrm{i}+1$ front $\}$ For each individual $\mathrm{p}$ in $\mathrm{Fi}$

Step 5: Increment the front counter $\mathrm{Fi}=\mathrm{Fi}+1$

Step 6: Set $\mathrm{Q}$ in the next front $\mathrm{Fi}=\mathrm{Q}$.

\subsection{Crowding distance calculation}

In addition to fitness value for each individual crowding distance is calculated. The crowding distance is calculated as:

Step 1: For each front Fi, in a non-dominated set I, 1 is the number of solution

Step 2: For all the individuals, initialize the distance to 0 i.e. $, F i(d j)=0, j$ corresponds to $j_{\text {th }}$ individual in Fi.And for each objective function $\mathrm{m}$

Step 3: Based on the objective $\mathrm{m}$ sort the individuals in front Fi (I=sort(Fi,m)).

Step 4: Boundary solution is assigned to infinite distance and for each individual in front $\mathrm{Fi}(\mathrm{I}(\mathrm{d} 1)=\infty$ and $\mathrm{I}(\mathrm{dl})=\infty)$. By this for all other points the boundary points are always selected. For $\mathrm{j}=2$ to (1-1)

Step 5: For $\mathrm{j}=2$ to (1-1)

- $I(d j)=I(d j)+(I(j+1) \cdot m-I(j-1 . m) / f m a x(m)-$ fmin $(\mathrm{m})$

- $I(j) m$ is the value of the $j$ th individual of the $m$ th objective function in $\mathrm{I} . \mathrm{f} \max (\mathrm{m})$ and $\mathrm{f} \min (\mathrm{m})$ are the maximum and minimum values of the $\mathrm{mth}$ objective function.

Based on the rank and crowding distance parents are selected from the population by using binary tournament selection.

\subsection{Crossover and Mutation}

Crossover is the mechanism by which design characteristics between any paired individuals are exchanged to form two new (child) individuals and single point crossover the data beyond the point in either organism string is swapped between the two parent organisms.

Mutation is a genetic operator used to maintain genetic diversity from one generation of a population of chromosomes to the next. The probability of mutation is given as $\mathrm{P}_{\mathrm{m}}$ and it is usually 100 times less than $\mathrm{P}_{c}$, where $\mathrm{P}_{\mathrm{c}}$ is the probability of crossover. 


\subsection{Generation of New Population}

The new population is filled up by taking the best individuals from the combined population based on rank and crowding distance.

\section{SIMULATION RESULTS}

Simulation results for permutations of condition in Table I.

A. Plot of Power optimization function that minimize the difference between the typical Power available (PT) and the Power consumed (Ptotal) by the current allocation scheme for 2 users

B. Plot of rate optimization function which is 1/(Minimum rate +1 ) for 2 users

C. Plot of Power optimization function which is minimizing the difference between the typical Power available (PT) and the Power consumed
(Ptotal) by the current allocation scheme for 4 users

D. Plot of rate optimization function which is 1/ (minimum rate +1 ), for 4 users

E. Plot of Power optimization function which is minimizing the difference between the typical Power available (PT) and the Power consumed (Ptotal) by the current allocation scheme for 8 users

F. Plot of rate optimization function which is $1 /($ minimum rate +1$)$, for 8 users

G. Plot of Power optimization function which is minimizing the difference between the typical Power available (PT) and the Power consumed (P total) by the current allocation scheme for 16 users H. Plot of rate optimization function which is $1 /($ minimum rate +1$)$ for 16 users

\section{TABLE I}

Maximum number of generations required for convergence for different set of experiments

\begin{tabular}{|c|c|c|c|c|c|c|c|c|c|}
\hline $\begin{array}{c}\text { S. } \\
\text { No }\end{array}$ & $\begin{array}{c}\text { Users } \\
(\mathbf{K})\end{array}$ & $\begin{array}{c}\text { Subcarriers } \\
(\mathbf{N})\end{array}$ & Population & $\begin{array}{c}\text { Maximum } \\
\text { generations }\end{array}$ & Pc & Pm & Dmax & $\begin{array}{c}\text { PT } \\
\text { (dB) }\end{array}$ & $\begin{array}{c}\text { Maximum } \\
\text { generation } \\
\text { for } \\
\text { convergence }\end{array}$ \\
\hline 1 & 2 & 64 & 50 fernatinal & 0.8 & 0.02 & 8 & 13 & 35 \\
\hline 2 & 4 & 64 & 30 & 100 & 0.8 & 0.02 & 16 & 24 & 28 \\
\hline 3 & 8 & 64 & 50 & 50 & 0.9 & 0.03 & 8 & 35 & 26 \\
\hline 4 & 16 & 64 & 30 & 50 & 0.9 & 0.03 & 8 & 56 & 25 \\
\hline
\end{tabular}

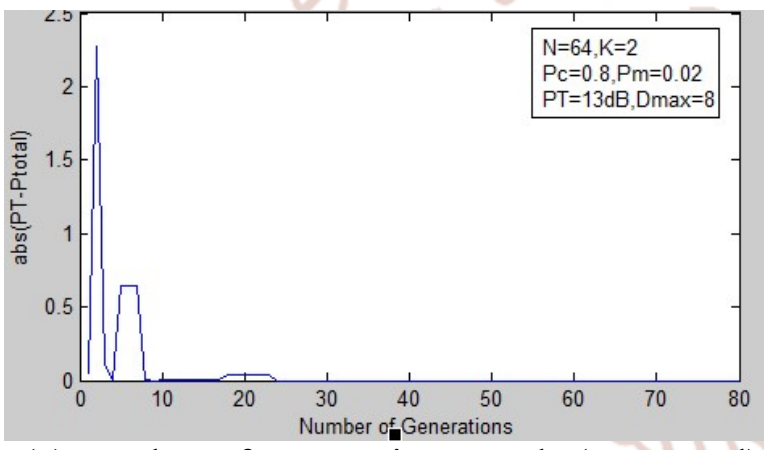

(a)Number of Generations vs abs(PT-Ptotal)

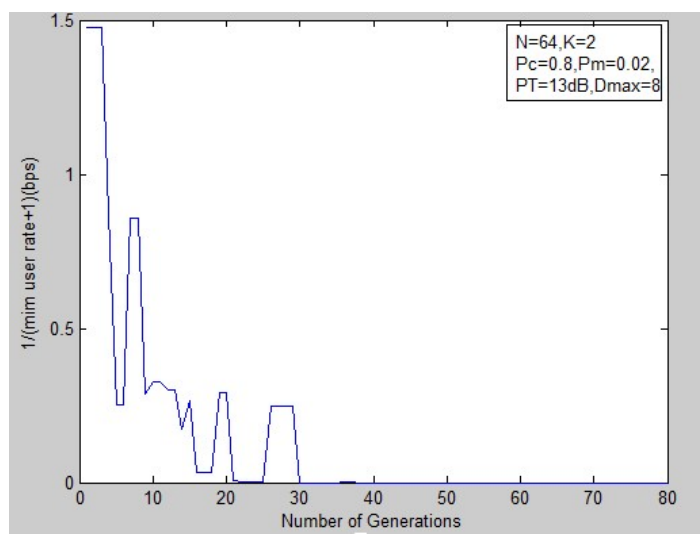

(b) Number of Generations vs $1 /$ (min user rate +1$)(b p s)$

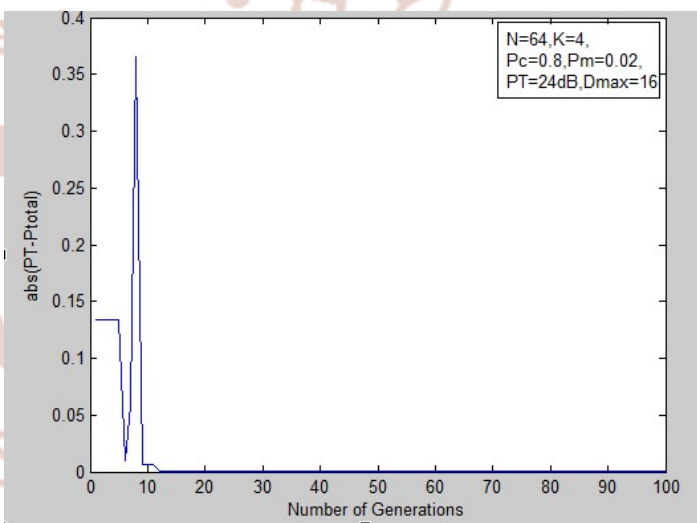

(c) Number of Generations vs abs(PT-Ptotal)

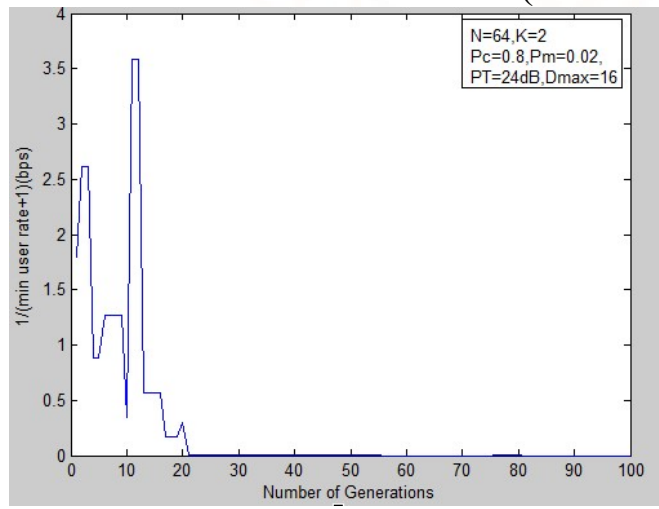

(d) Number of Generations vs $1 /$ (min user rate +1$)(\mathrm{bps})$ 


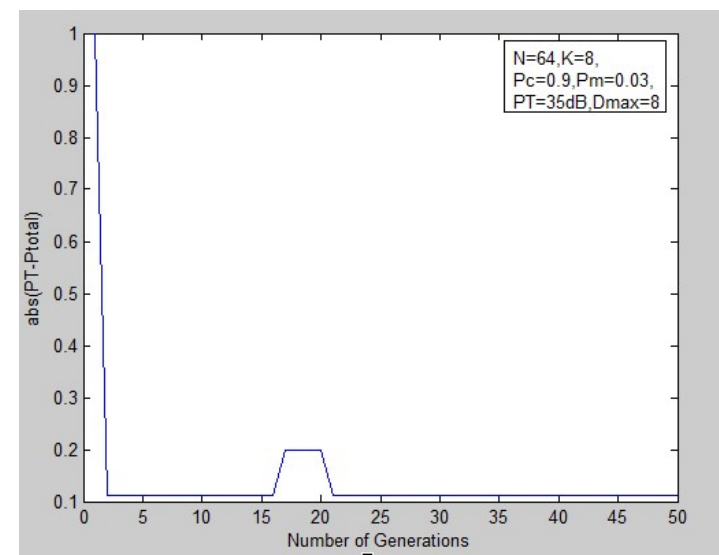

(e) Number of Generations vs abs(PT-Ptotal)

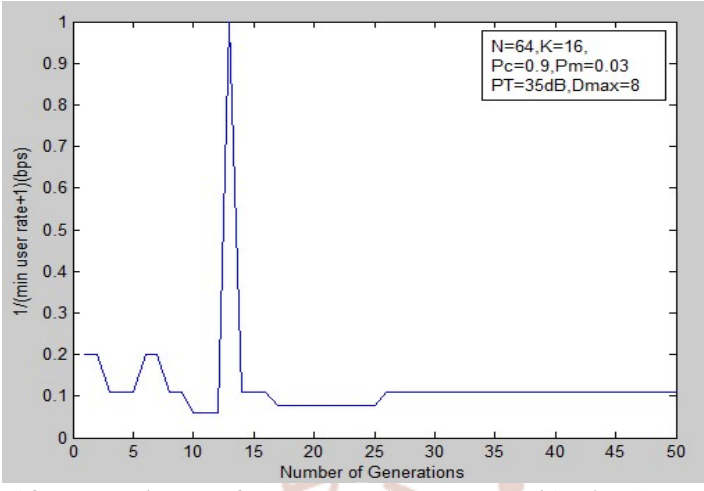

(f) Number of Generations vs $1 /$ (min user rate +1$)(\mathrm{bps})$

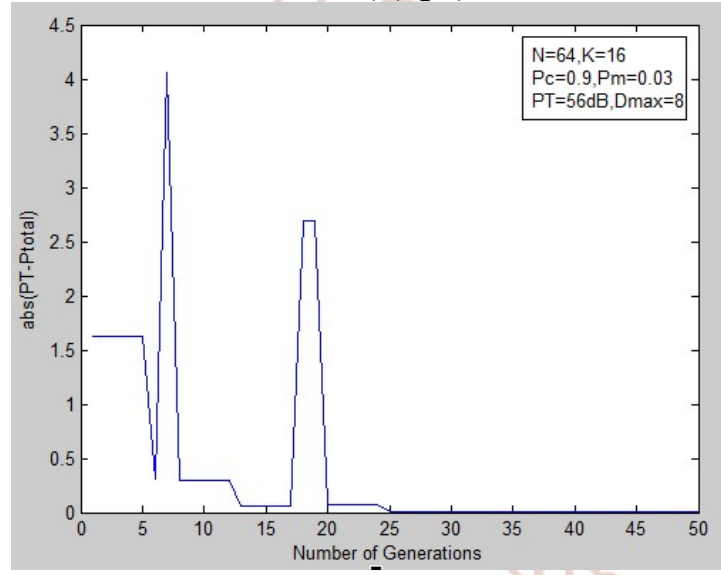

(g) Number of Generations vs abs(PT-Ptotal)

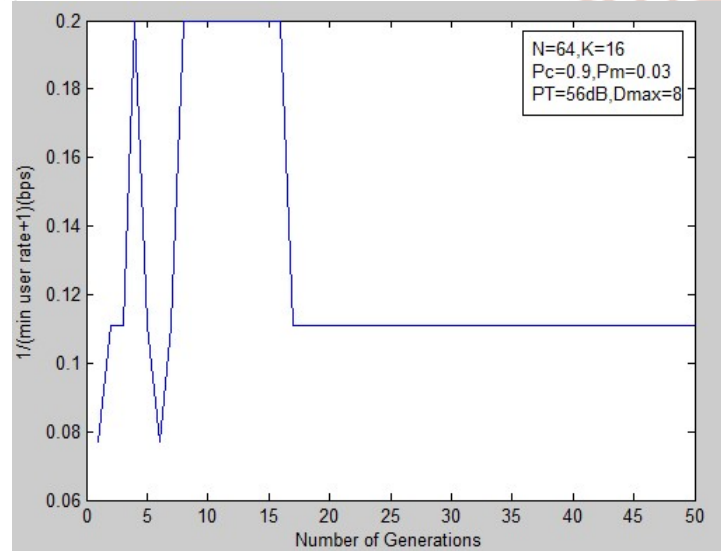

(h) Number of Generations vs 1/(min user rate +1$)($ bps $)$

Fig1. Simulation results for permutations of conditions given in Table I

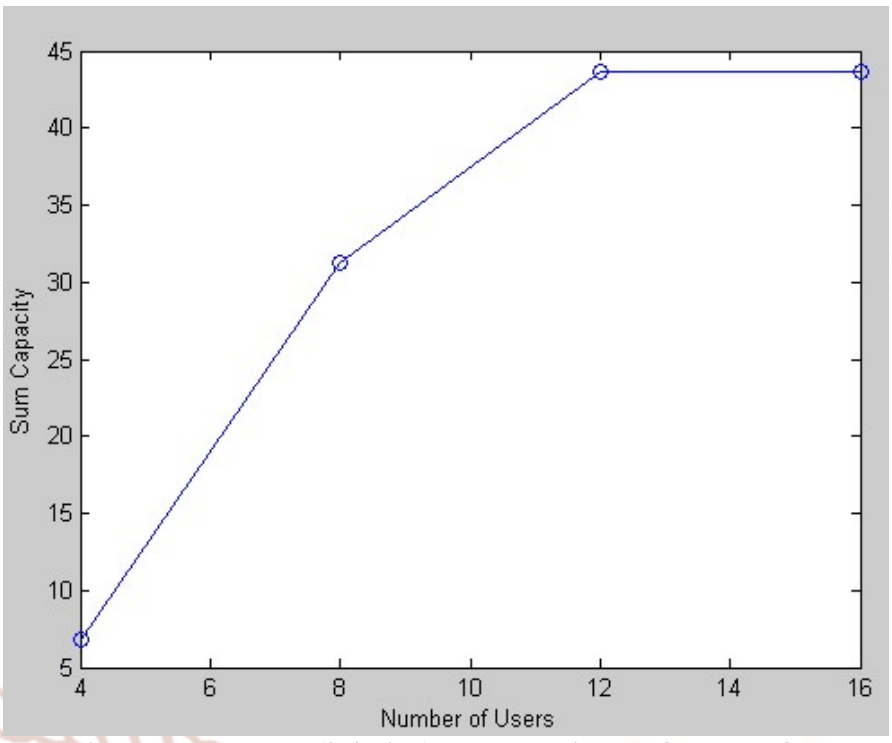

Fig 2 sum rate $(\mathrm{b} / \mathrm{s} \mathrm{hz})$ vs number of users for $\mathrm{snr}=24.6 \mathrm{db}$

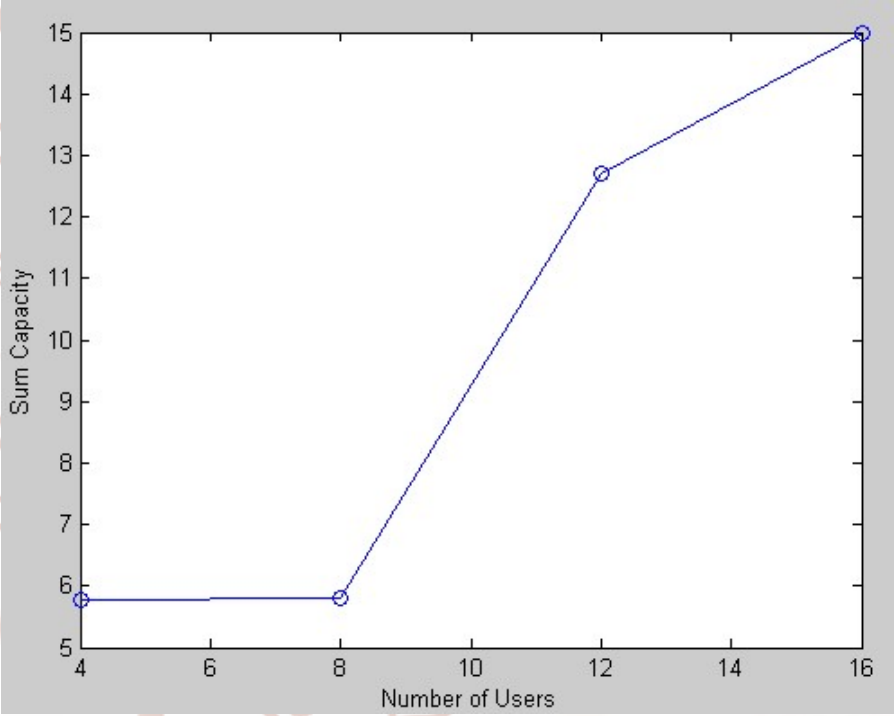

Fig3. Sum rate (b/s hz) vs number of users for $\mathrm{snr}=4.6 \mathrm{db}$

The Simulation results from FIG 2 and FIG 3 indicate the optimized Sum rates $(\mathrm{b} / \mathrm{s} / \mathrm{Hz})$ for fixed number of sub-carriers $(\mathrm{N}=64)$ versus number of users $(\mathrm{K}=4,8,16)$ for $\mathrm{SNR}=24.6 \mathrm{Db}$ (Dmax=16 bits) (Fig 2) and for $\mathrm{SNR}=4.6 \mathrm{db}\left(\mathrm{D}_{\max }=8 \mathrm{bits}\right)($ Fig 3$)$.

\section{CONCLUSION}

NSGA-II has been proposed for subcarrier allocation in MIMO-OFDM system for the multiuser and simultaneous bit-loading. The solution generated by the algorithm is found to be suitable for different sets of users and subcarriers taking multiple conflicting objectives into the algorithm and it guarantees fairness among the users by means of optimizing the rate. 


\section{REFERENCES}

1. Nitin Sharma, K. R. Anupama (2014) "On the use of NSGA-II for multi-objective resource allocation in MIMO-OFDMA systems". Wireless Network17 (5): 1191-1201.

2. Nitin Sharma, Dr K R Anupama, (2014) “A novel Genetic Algorithm for Adaptive Resource Allocation in MIMO-OFDM Systems with Proportional Rate Constraint". Wireless Personal Communications Volume 61, Number 1, 113-128.

3. Wong,C.Y., Cheng, R. S., Letaief, K. B., \& Murch, R. D (1999). "Multiuser OFDM with adaptive subcarrier and power allocation" .IEEE Journal of Selected Areas in Communication, 17(10), 1747-1758.

4. Rhee, W. \& Cioffi, J. M. (2008). "Increasing in capacity of multiuser OFDM system using dynamic sub channel allocation". In Proceedings of IEEE international vehicular technology conference (Vol. 2, pp. 1085-1089).

5. Jang, J., \& Lee, K. B. (2007). "Transmit power adaptation for multiuser OFDM system". IEEE Journal of Selected Areas in Communication, 21, 171-178.

6. Shen, Z., Andrews, J. G., \& Evans, B. L. (2009). "Adaptive resource allocation in multiuser OFDM systems with proportional rate constraints". IEEE Transactions on Wireless Communications, 4(6), 2726-2737.

7. Kim, I., Park, I.-S., \& Lee, Y. H. (2010). Use of linear programming for dynamic subcarrier and bit allocation in multiuser OFDM. IEEE Transactions on Vehicular Technology, 55(4), 1195-1207.

8. Y. B. Reddy, (2011) "Genetic Algorithm Approach for Adaptive Subcarrier, Bit, and Power Allocation" Proceedings of the 2011 IEEE International Conference in Networking, Sensing and Control, London, UK, 15-17.

9. Kalyanmoy, D., Amrit, P., Sameer, A., \& Meyrivan, T. (2005). "A fast and elitist multi objective genetic algorithm: NSGA-II".IEEE Transactions on Evolutionary Computation, 6(2), 182-197.

10. Xiao, X., Hu, Z., Zhu, G. \& Li, L. (2008). "Adaptive subcarrier allocation for increasing the capacity of multiuser spatial multiplexing based OFDM systems". In Proceedings of IEEE PIMRC, Berlin, Germany (pp. 377-381).

11. Andersen, J. B. (2004). "Array gain and capacity far known random channels with multiple elements arrays at both ends". IEEE Journal of Selected Areas in the field of Communication, 18(2), 2172-2178.

12. .Kalyanmoy, Deb(2003)."Multi-objective rate and power optimization by evolutionary algorithms (1st ed.)". USA: Wiley.

13. Nitin Sharma, Rao. A, Dewan. A, Safdari, M "Rate adaptive resource allocation for multiuser OFDM using NSGA-II Proceeding of Fourth IEEE Conference on Wireless Communication and Sensor Networks" (WCSN-2008). 